\title{
Survival data from PALOMA-3 reported
}

The combination of the cyclin-dependent kinase 4/6 inhibitor palbociclib plus fulvestrant was approved by the FDA for women with hormone receptor-positive, HER2-negative advanced-stage or metastatic breast cancer in 2016, on the basis of a significant increase in progression-free survival (PFS) versus placebo plus fulvestrant observed in the PALOMA-3 trial. Newly published data reveal the overall survival (OS) outcomes of patients in this cohort.

At a median follow-up duration of 44.8 months, patients receiving palbociclib plus fulvestrant had a median OS duration of 34.9 months versus 28.0 months in the placebo plus fulvestrant group (HR 0.81 , 95\% CI 0.64-1.03; $P=0.09$ ). These results, although not statistically significant, support the previous observation of an improvement in PFS of 6.6 months reported in 2016.

The investigators attributed the lack of a significant improvement in OS in part to the commercial availability of palbociclib, which $18 \%$ of patients who had disease progression on placebo subsequently received.

Patients receiving palbociclib plus fulvestrant had a similar profile of adverse events to those reported in the previous analysis, including grade $\geq 3$ neutropenia in $69.6 \%$ and grade $\geq 3$ leukopenia in $38.3 \%$.

Prespecified subgroup analyses demonstrated that women who remained sensitive to the previous line of endocrine therapy derived the highest level of benefit from palbociclib; whereas women with intrinsically endocrine resistant disease were less likely to benefit.

These findings confirm that the beneficial improvements in PFS duration are, to an extent, retained as improvements in OS with longer follow-up monitoring. However, as noted by the authors, owing to difficulties in investigating effects on OS in a cohort of patients who are likely to survive, and receive other treatments for a duration that exceeds the trial follow-up period, data from a larger cohort of patients, or from other sources, are likely to be required to demonstrate a statistically significant improvement in OS.

Peter Sidaway

ORIGINAL ARTICLE Turner, N. C. et al. Overall survival with palbociclib and fulvestrant in advanced breast cancer. N. Engl. J.Med. https://doi.org/10.1056/NEJMoa1810527 (2018)

\section{Olaparib maintenance moves to first line}

Women with newly diagnosed advanced-stage ovarian cancer have a high risk of disease relapse within 3 years of treatment.

Now, newly published data from the SOLO1 study demonstrate that progression-free survival (PFS) can be prolonged substantially by using the poly(ADP-ribose) polymerase (PARP) inhibitor olaparib in patients with a germline or somatic BRCA1/2 mutation.

A total of 391 patients were randomly assigned (2:1) to receive either olaparib maintenance therapy or placebo until disease progression, following a complete or partial response to platinum-based therapy. At a median follow-up duration of 40.7 months, $60 \%$ of patients receiving olaparib achieved the primary end point of PFS versus $27 \%$ of patients in the placebo group (HR $0.30,95 \% \mathrm{Cl} 0.23-0.41 ; P<0.001$ ). As part of the trial design, patients with no evidence of disease at 2 years ceased olaparib maintenance therapy at this time point; many of these patients remained free of any detectable disease following olaparib cessation.

In total, $39 \%$ of patients in the olaparib group had grade $3-4$ adverse events versus $18 \%$ in the placebo group. Notable increases in anaemia
( $22 \%$ versus $2 \%$ ) and neutropenia ( $9 \%$ versus $5 \%$ ) were reported. The incidence of de novo acute myeloid leukaemia (1\%) was consistent with that reported in other trials investigating the effects of PARP inhibitors. In an analysis of health-related quality of life, patients in the placebo group had a greater reduction in Trial Outcome Index Score than those in the olaparib group (estimated between-group difference -3.00 points, $95 \% \mathrm{Cl}-4.78$ to -1.22 ), although this difference was not clinically meaningful.

These findings demonstrate that robust improvements in PFS can be obtained using olaparib in the first-line maintenance setting. The authors also note the potential of this approach to be curative in a subset of patients - as observed in previous trials involving patients with relapsed disease; however, longer follow-up monitoring will be required in order to identify this subset.

Peter Sidaway

ORIGINAL ARTICLE Moore, K. et al. Maintenance olaparib in patients with newly diagnosed advanced ovarian cancer. N. Engl.J.Med.https://doi.org/10.1056/NEJMoa1810858 (2018)

\section{LUNG CANCER}

\section{From WCLC 2018}

The World Conference on Lung Cancer (WCLC) was recently held in Toronto. Three of the studies selected as 'top 5' abstracts in this conference were published in the New England Journal of Medicine.

In the PACIFIC trial, the efficacy and safety of durvalumab are being tested in patients with locally advanced, unresectable non-small-cell lung cancer (NSCLC) and good performance status after chemoradiotherapy. An analysis of the second primary end point, overall survival (OS), was presented: 24-month OS was $66.3 \%$ and $55.6 \%$ with durvalumab and placebo, respectively $(P=0.0025)$. Grade 3-4 adverse events (AEs) occurred in $30.5 \%$ and $26.1 \%$ of patients, respectively, leading to treatment discontinuation rates of $15.4 \%$ and $9.8 \%$.

Brigatinib is compared with crizotinib as a first-line treatment for patients with ALK-rearranged advanced-stage NSCLC in the ALTA-1L trial. At a median follow-up duration $<12$ months, the objective response rate was higher with brigatinib: $71 \%$ versus $60 \%$. The confirmed rates of intracranial response were $78 \%$ and $29 \%$, respectively. The incidence of grade 3-5 AEs was also higher in the brigatinib group ( $61 \%$ versus $55 \%)$.

In IMpower133, first-line carboplatin and etoposide were combined with atezolizumab or placebo as a first-line treatment for patients with extensive-stage small-cell lung cancer. At 13.9 months, median OS durations were 12.3 months with atezolizumab and 10.3 months with placebo $(P=0.007)$.

The incidence of AEs of any grade was similar (94.9\% versus $92.3 \%)$.

The other two studies were LUME-Meso, testing the addition of nintedanib versus placebo to standard-of-care chemotherapy in patients with unresectable malignant pleural mesothelioma, and the NELSON trial, comparing $\mathrm{CT}$ screening with no screening in asymptomatic individuals deemed at high risk for lung cancer. Promising results have been obtained in both studies, which, to our knowledge, have not yet been published.

Diana Romero

ORIGINAL ARTICLES Antonia, S. J. et al. Overall survival with durvalumab after chemoradiotherapy in stage III NSCLC. N. Engl.J. Med. https://doi.org/10.1056/NEJMoa1809697 (2018)| Camidge, D. R. et al. Brigatinib versus crizotinib in ALK-positive non-small-cell lung cancer. N. Engl.J. Med. https://doi.org/ 10.1056/NEJMoa1810171 (2018) | Horn, L. et al. First-line atezolizumab plus chemotherapy in extensive-stage small-cell lung cancer. N. Engl.J. Med. https://doi.org/10.1056/ NEJMoa1809064 (2018) 